\title{
Inescapable shock interferes with the acquisition of a low-activity response in an appetitive context
}

\author{
ROBERT A. ROSELLINI and JOSEPH P. DeCOLA \\ State University of New York, Albany, New York 12222
}

\begin{abstract}
This study investigates the effects of exposure to inescapable shock on the acquisition of a low-activity appetitive response using a trial procedure. Inescapable shock was found to interfere with the acquisition of a nose-poke response to obtain food as compared with animals exposed to either escapable shock or no shock. In addition, general activity levels were measured separately during the trial and the intertrial interval during the appetitive test. Inescapably shocked animals were less active during the trial component than were either the escapably shocked or the nonshocked animals. However, no differential levels of activity were observed during the intertrial interval component of the appetitive test. The relevance of these findings for both the learned helplessness and the learned inactivity hypotheses is discussed.
\end{abstract}

Exposure to inescapable shock produces strong interference with an organism's subsequent ability to learn a novel response to escape shock (Maier, Albin, \& Testa, 1973; Overmier \& Seligman, 1967; Seligman \& Beagley, 1975). However, animals given an equivalent amount of escapable shock or no shock typically acquire the novel escape response. Maier and Seligman (1976) have proposed that this learned helplessness phenomenon results from the animal's acquiring the expectancy, during the exposure to inescapable shock, that responding and outcomes are independent. This expectancy is held to reduce the incentive for initiating responding (i.e., the motivational deficit) and to interfere proactively with learning that the outcome is now contingent on responding (i.e., the associative deficit). Thus, the animal exposed to inescapable shock subsequently fails to learn the response to terminate shock or learns it more slowly than do controls.

This learned helplessness hypothesis suggests that the animal acquires a general expectancy about the consequences of its behavior that may generalize to a wide variety of contexts. Recent research has demonstrated the generality of the learned helplessness phenomenon. The phenomenon transfers across different aversive training and testing contexts. Altenor, Kay, and Richter (1977) and Braud, Wepmann, and Russo (1969) found that inescapable shock interferes with learning an escape response in a cold water maze. Rosellini and Seligman (1975) have reported that inescapable shock interferes with the

This research was supported by NIMH Grant 1-R03-MH31424 and NSF Grant BNS-7820678. We wish to thank John Lee for assistance in execution of this work. Requests for reprints should be sent to the first author, Department of Psychology, SUNY at Albany, Albany, New York 12222. acquisition of a response to escape from primary frustration. The detrimental effects of exposure to uncontrollable events is not limited to aversive contexts. Rosellini (1978) has found that inescapable shock results in retarded acquisition of a response to obtain food. Furthermore, Goodkin (1976) has reported that noncontingent delivery of food to hungry animals interferes with the subsequent acquisition of a response to escape/avoid electric shock.

These demonstrations of the motivational generality of effects produced by prior uncontrollable events are congruent with the associative deficit postulated by the learned helplessness hypothesis. However, these studies do not provide exclusive support for this position. Alternative accounts such as learned inactivity (Glazer \& Weiss, 1976a), competing response (Bracewell \& Black, 1974), and neurochemical mediation (Anisman, 1975) may also be congruent with the generality of the phenomenon. The learned inactivity hypothesis may propose slower response acquisition during an appetitive test, following exposure to inescapable shock, due to the animal's prior learning to be inactive. This inactivity could transfer to the novel test context, which typically requires an active response (i.e., leverpressing, swimming, or shuttling), resulting in a deficit in acquisition. These alternative hypotheses rely on the animal's inability to emit active responses either because the animal learns to be inactive (Glazer \& Weiss, 1976a) or because of neurochemical changes resulting in reduced activity (Anisman, 1975). Therefore, the generalization of the deleterious effects of inescapable shock can be viewed as the direct transfer of inactivity to the test context.

The primary purpose of the present study was to determine whether the generality of the learned help- 
lessness phenomenon can be accounted for by the direct transfer of inactivity to an appetitive test. Glazer and Weiss (1976b) demonstrated that animals exposed to inescapable shock fail to exhibit a deficit in learning a low-activity response to escape/avoid shock. In fact, they showed that inescapable shock produces faster acquisition of the low-activity nosepoke response than does exposure to either escapable shock or no shock. These findings and the learned inactivity hypothesis strongly suggest that inescapable shock will not interfere with, and indeed may facilitate, the subsequent acquisition of a low-activity nose-poke response. Thus, in the present study, we employed a nose-poke response to test for any deficits in the acquisition of this low-activity response in an appetitive context.

A second purpose of the present study was to provide direct measures of general activity levels during the appetitive test. Anisman, deCatanzaro, \& Remington (1978) and Jackson, Maier, and Rappaport (1978), using an aversive test, found lower activity levels in animals that had received inescapable shock than they found in nonshocked controls. However, none of the studies investigating the motivational generality of learned helplessness obtained direct measures of activity. Thus, it is important to determine whether changes in activity are general or specific to contexts employing shock. Direct activity measures would aid in removing much of the post hoc flavor of these alternative hypotheses when applied to nonaversive test contexts.

\section{METHOD}

\section{Subjects}

The subjects were 24 male Holtzman rats, approximately 110 days of age at the start of the experiment. One week prior to the experimental treatment, all rats were placed on an $85 \%$. body-weight deprivation schedule. They were maintained on adlib water and were run during the light phase of a 12 -h dark/light cycle.

\section{Apparatus}

Three experimental chambers were used for shock training. Each chamber was $45.7 \mathrm{~cm}$ long $\times 24.5 \mathrm{~cm}$ high $\times 21.6 \mathrm{~cm}$ wide. The side walls were constructed of aluminum, and the ceiling was constructed of clear Plexiglas. The floor was constructed of stainless steel rods $6.36 \mathrm{~mm}$ in diameter and spaced $19.05 \mathrm{~mm}$ apart. The chamber was divided in half by an aluminum wall that had a $10.8 \mathrm{~cm}$ high $\times 6.35 \mathrm{~cm}$ wide opening in the center at floor level. A cue light was centered $20.3 \mathrm{~cm}$ above the grid floor on each end wall of the chamber. Each chamber had one photocell located $4.5 \mathrm{~cm}$ above the grid floor and $8 \mathrm{~cm}$ from the end wall. Shock was delivered to each chamber by a Coulbourn solid state shock source (Model E13-16). Each chamber was housed in a sound-and light-attenuating container that was equipped with a $28-\mathrm{V}$ houselight, a speaker for delivery of white noise, and a ventilating fan.

Three different chambers were used for testing. These chambers were modified Lafayette activity platforms (Model 86010). Each test chamber was $29.85 \mathrm{~cm}$ long $\times 29.85 \mathrm{~cm}$ high $\times 30.48 \mathrm{~cm}$ wide. A food cup $(44 \times 31.75 \times 12.7 \mathrm{~mm})$ was mounted on the front wall $38 \mathrm{~mm}$ above the solid floor and $7.6 \mathrm{~cm}$ from the side wall. The front wall had a $19-\mathrm{mm}$-diam hole $15.24 \mathrm{~cm}$ to the left of the food cup and at the same elevation above the floor. This hole allowed the animal access to the response manipulandum, which was a metal plate resting $6.35 \mathrm{~mm}$ behind the hole. Contact with the metal plate, which was detected by means of a touch-sensitive circuit, was defined as the response. A 28-V dc light was mounted directly above this plate and served as the trial signal.

\section{Procedure}

Shock training. On the 1st day of the experiment, the three experimental groups were given differential shock training. The first group $(\mathrm{N}=8)$ was trained to escape shock (Group E). Training consisted of one session of 80 trials of up to $30 \mathrm{sec}$ of $.90-\mathrm{mA}$ shock. For the first five trials, shock could be terminated by the animal's crossing from one side of the shuttlebox to the other (FR 1). On the remaining 75 trials, an FR 2 contingency was in effect whereby the animals were required to cross from one side to the other and then back to the original side. Escape trials were presented on a variable time 60 -sec schedule (range $5-115 \mathrm{sec}$ ). The training session was therefore approximately $90 \mathrm{~min}$ long. Each animal in the second group (Group I) was yoked to an animal in the escape group. Thus, each pair of animals received an identical pattern and duration of shock. However, the shock for Group I animals was inescapable, since its termination was not contingent on their behavior but on the behavior of the Group $\mathrm{E}$ animals. The animals in the third group (Group C) were used as controls. They were placed in the training chambers for a yoked duration of time but were not exposed to electric shock.

Appetitive test. Twenty-four hours following shock training, the appetitive test was started. Each test session consisted of 50 trials during which a $45-\mathrm{mg}$ Noyes food pellet could be obtained on a CRF schedule by the animal nose-poking into the access hole and contacting the response sensor. The trials were signaled by illumination of the cue light behind the access hole. If an animal failed to respond within $20 \mathrm{sec}$ of the signal onset, the signal was turned off and the trial was terminated without pellet delivery. The occurrence of a response prior to automatic trial termination would produce delivery of one pellet and terminate the cue light. The intertrial interval was $30 \mathrm{sec}$. Testing was conducted for six sessions. Two main dependent measures were employed in this study. The first was the latency to respond; the second was the amount of activity emitted during each session, which was monitored by means of the activity platforms.

\section{RESULTS}

All rats in Group E learned the FR 2 shuttle response to terminate shock. No failures to escape shock were observed for any of these animals on the last 20 trials of training. The mean amount of shock received by this group-and, therefore, by Group Iwas $5.04 \mathrm{sec}(\mathrm{SD}=3.24)$.

\section{Response Latency}

Animals exposed to inescapable shock showed retarded acquisition of the nose-poke response to obtain food as compared with the animals exposed to either escapable shock or no shock. Figure 1 shows the mean response latencies for the three treatment groups for each of the six test sessions. In general, all groups show a typical learning curve as indicated by decreasing response latencies across sessions. More importantly, there is a tendency for Group I animals to show a pattern of decreasing latencies different from either Group E or Group C.

Planned comparisons of these response latencies supported the impression that Group I generally had 
longer latencies than either Group $\mathrm{E}$ or Group $\mathrm{C}$ $[F(1,21)=6.18, p<.05]$, while Groups $E$ and $C$ did not differ $[F(1,21)<1.0]$.

\section{Activity}

Since trial duration was under the subject's control, the activity measure was transformed to activity per second. As can be seen in Figure 2, large differences in trial and intertrial interval activity rate emerged across sessions for all treatment groups. This pattern resulted in a significant trial $\times$ session interaction $[F(5,105)=6.36, p<.001]$. Separate analyses of the activity measure for the trial and intertrial interval were conducted as a function of groups (3) and session (6). A marginal significant increase in intertrial interval activity was observed across the six test sessions $[F(5,105)=2.27, p=.057]$. However, no main group differences or interactions of groups with sessions were observed (all ps $>.20$ ).

Large increases in activity rate were observed during the trial as a function of sessions, thereby resulting in a significant sessions effect $[F(5,105)=8.98$, $\mathrm{p}<.001]$. In addition, the treatment groups tended to show differential increases in activity across the six test sessions. This pattern resulted in a marginally re-

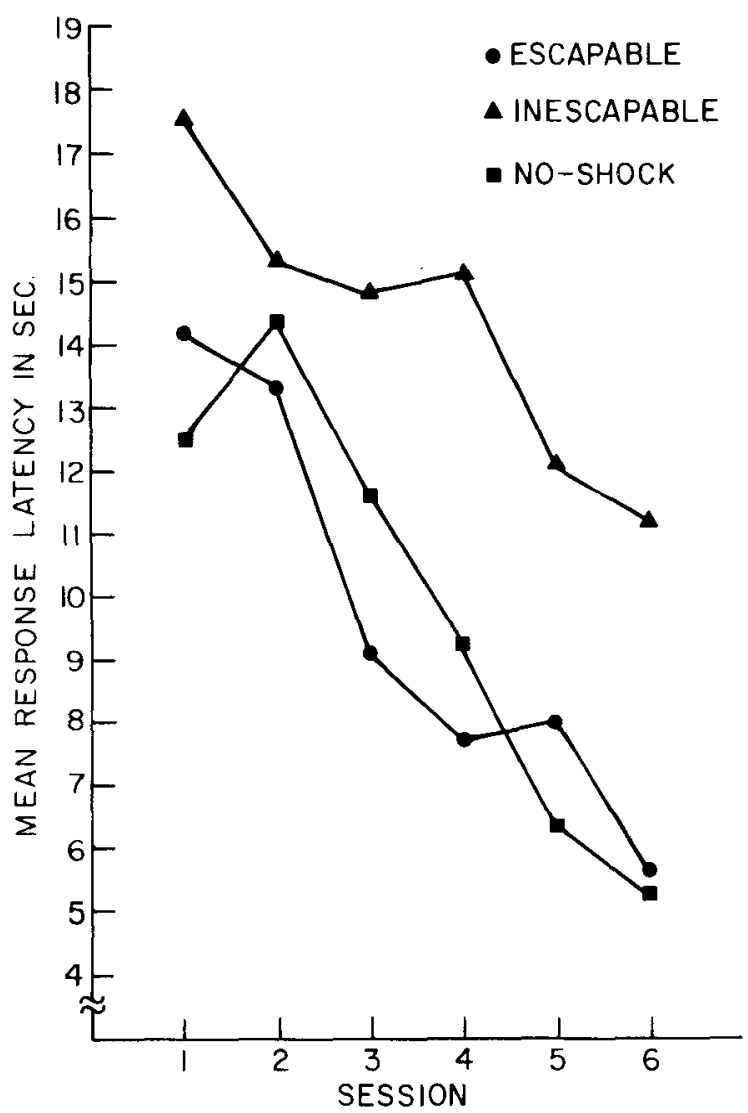

Figure 1. Mean nose-poke latencies for the three treatment groups on the six test sessions.

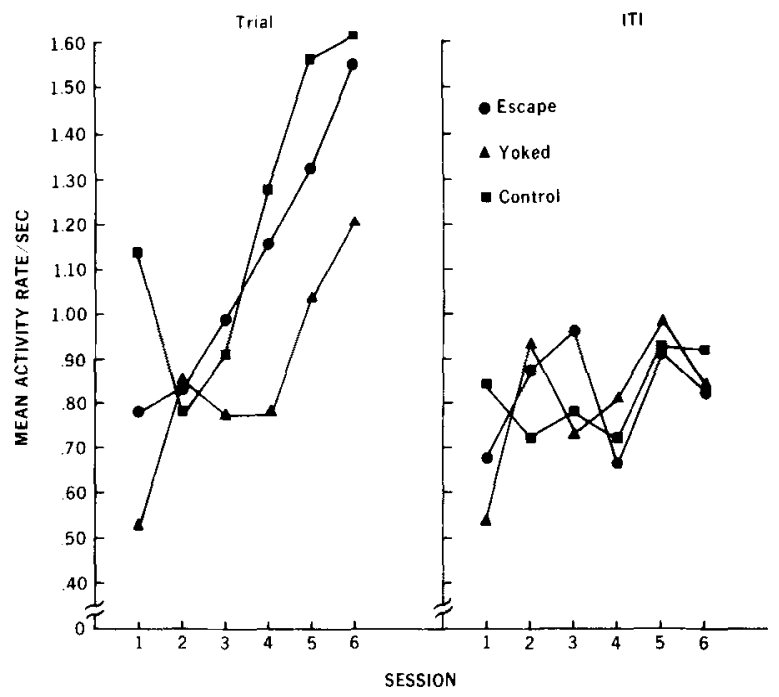

Figure 2. Mean activity rates for the three treatment groups during the six test sessions for the trial (left panel) and the intertrial interval (right panel).

liable cubic component for the groups $\times$ session interaction $[F(2,21)=3.32, p=.056]$. Further analysis of this trend showed Group I to be marginally less active $[F(1,21)=3.65, .05<p<.10]$ than both Groups $E$ and $C$, which did not differ from each other $(p>.10)$.

\section{DISCUSSION}

Exposure to inescapable shock results in a deficit in the acquisition of a response to obtain food, even when the operant response is one that presumably requires little physical activity. Previous research has demonstrated that inescapable shock produces a deficit in the subsequent acquisition of a more active response, such as leverpressing (cf. Rosellini, 1978). These findings, taken together, suggest that the activity requirement of the response during the appetitive test may not be the deterimining factor in the cross-motivational effects of prior exposure to inescapable shock. This is not consistent with the learned inactivity hypothesis, which suggests that inescapable shock should facilitate, rather than interfere with, the acquisition of a nose-poke response, which presumably requires little activity. It should be noted that, while a nose-poke response was used in both the present experiment and in the Glazer and Weiss (1976a, 1976b) studies, we employed an operant chamber, whereas they used a restraint apparatus for the test phase. Thus, relatively more activity may have been required in our test context than in that employed by Glazer and Weiss. It may be tempting to speculate that such a potentially different activity requirement may account for the differential results of these studies. However, this does not appear to be a viable account since, as discussed below, we observed no 
differential levels of activity during the intertrial interval as a function of exposure to inescapable shock.

Currently, there is little doubt that inescapable shock can produce lower activity when the animal is reexposed to either shock (Jackson, Maier, \& Coon, 1979) or the context in which shock was originally delivered (MacLennan, Jackson, \& Maier, 1980). In agreement with these studies, we also observed lower activity levels in inescapably shocked animals than in controls. Maier and Jackson (1979) cogently argue that decreases in activity following exposure to inescapable shock may be mediated by a conditioned analgesic response. The present study did not incorporate either of the above procedural conditions. Hence, these processes mediating the reduced activity in shock contexts presumably should not be involved, since testing was performed in a context different from training and shock was not employed.

The activity measure demonstrates that inescapably shocked animals tend to be less active during the trial than do animals exposed to either escapable shock or no shock. No differences in activity between the groups are apparent during the intertrial interval. The former differences appear to be congruent with activity hypotheses attempting to account for the cross-motivational generality of the learned helplessness effect. However, it would appear difficult for such a position to explain the lack of differences in intertrial interval activity between shock groups. Presumably, an activity deficit that is sufficiently severe to prevent an animal from nose-poking to gain food reinforcement should be evidenced in intertrial interval activity. This pattern of results suggests that activity differences within a trial may result from, and not be the cause of, disparate rates of response acquisition in the different shock groups.

The lower trial activity of the yoked group may be explained more directly from the associative deficit postulated by the learned helplessness hypothesis (Maier \& Seligman, 1976). Animals in the yoked group exhibiting a deficit in learning the responsereinforcer contingency during the trial would also, of necessity, have less of an opportunity to acquire the stimulus-reinforcer contingency. That is, if an animal fails to respond on a trial, it is effectively presented with the conditional stimulus (i.e., discriminative stimulus), which is not followed by the reinforcer. The trial CS presumably comes to control activity directed at either the response manipulandum or the food cup. Thus, animals preexposed to inescapable shock would be expected to be less active during the trial, since the CS has accrued less strength by virtue of the slower acquisition of the responsereinforcer contingency. This view also suggests that no differences in activity between the groups should be observed during the intertrial interval since the trial CS is absent, and this was found to be the case.

\section{REFERENCES}

Altenor, A., Kay, E., \& Richter, M. The generality of learned helplessness in the rat. Learning and Motivation, 1977, 8, 54-61.

Anisman, H. Time-dependent variations in aversively motivated behaviors: Nonassociative effects of cholinergic and catecholaminergic activity. Psychological Review, 1975, 82, 359-385.

Anisman, H., deCatanzaro, D., \& Remington, C. Escape performance deficits following exposure to inescapable shock: Deficits in motor response maintenance. Journal of Experimental Psychology: Animal Behavior Processes, 1978, 4, 197-218.

Brace Well, R. J., \& BlaCK, A. H. The effect of restraint and noncontingent preshock on subsequent escape learning in the rat. Learning and Motivation, 1974, 5, 53-69.

Braud, W., Wepmann, B., \& Russo, D. Task and species generality of the learned helplessness phenomenon. Psychonomic Science, 1969, 16, 154-155.

Glaze R, H. I., \& WEISS, J. M. Long-term and transitory interference effects. Journal of Experimental Psychology: Animal Behavior Processes, 1976, 2, 191-201. (a)

Glazer, H. I., \& Weiss, J. M. Long-term interference effect: An alternative to "learned helplessness." Journal of Experimental Psychology: Animal Behavior Processes, 1976, 2, 202-213. (b)

Goodkin, F. Rats learn the relationship between responding and environmental events: An expansion of the learned helplessness hypothesis. Learning and Motivation, 1976, 7, 382-393.

Jackson, R. L., Maier, S. F., \& Coon, D. J. Long-term analgesic effects of inescapable shock and learned helplessness. Science, 1979, 206, 91-93.

Jackson, R. L., Maier, S. F., \& Rapaport, P. M. Exposure to inescapable shock produces both activity and associative deficits in the rat. Learning and Motivation, 1978, 9, 69-98.

Maclennan, A. J., Jackson, R. L., \& Maier, S. F. Conditioned analgesia in the rat. Bulletin of the Psychonomic Society, $1980,15,387-390$.

Maier, S. F., Albin, R. W., \& Testa, T. J. Failure to learn to escape in rats previously exposed to inescapable shock depends on the nature of the escape response. Journal of Comparative and Physiological Psychology, 1973, 85, 581-592.

MAIER, S. F., \& JACKson, R. L. Learned helplessness: All of us were right (and wrong): Inescapable shock has multiple effects. In G. H. Bower (Ed.), The psychology of learning and motivation. New York: Academic Press, 1979.

Maier, S. F., \& Seligman, M. E. P. Learned helplessness: Theory and evidence. Journal of Experimental Psychology: General, 1976, 105, 3-46.

Overmier, J. B., \& Seligman, M. E. P. Effects of inescapable shock upon subsequent escape and avoidance behavior. Journal of Comparative and Physiological Psychology, 1967, 63, 23-33.

Rosellini, R. A. Inescapable shock interferes with the acquisition of a free appetitive operant. Animal Learning \& Behavior, $1978,6,155-159$.

Roseldini, R. A., \& Seligman, M. E. P. Frustration and learned helplessness. Journal of Experimental Psychology: Animal Behavior Processes, 1975, 1, 149-157.

Seligman, M. E. P., \& Beagley, G. Learned helplessness in the rat. Journal of Comparative and Physiological Psychology, $1975,88,534-542$.

(Manuscript received January 20, 1981; revision accepted for publication May 3, 1981.) 